\title{
\begin{tabular}{l|l} 
MitTraries & DSpace@MIT
\end{tabular}
}

\author{
MIT Open Access Articles
}

\section{Effect of temperature on superconducting nanowire single-photon detector noise}

The MIT Faculty has made this article openly available. Please share how this access benefits you. Your story matters.

Citation: Bahgat Shehata, A., A. Ruggeri, F. Stellari, Alan J. Weger, P. Song, K. Sunter, F. Najafi, Karl K. Berggren, and Vikas Anant. "Effect of Temperature on Superconducting Nanowire SinglePhoton Detector Noise." Edited by Manijeh Razeghi, Dorota S. Temple, and Gail J. Brown. Optical Sensing, Imaging, and Photon Counting: Nanostructured Devices and Applications (August 28, 2015).

As Published: http://dx.doi.org/10.1117/12.2188234

Publisher: SPIE

Persistent URL: http://hdl.handle.net/1721.1/110750

Version: Final published version: final published article, as it appeared in a journal, conference proceedings, or other formally published context

Terms of Use: Article is made available in accordance with the publisher's policy and may be subject to US copyright law. Please refer to the publisher's site for terms of use. 


\title{
Effect of temperature on Superconducting Nanowire Single-Photon Detector noise
}

\author{
A. Bahgat Shehata*a , A. Ruggeri ${ }^{\dagger a}$, F. Stellari ${ }^{\mathrm{a}}$, Alan J. Weger ${ }^{\mathrm{a}}$, P. Song ${ }^{\mathrm{a}}$, \\ K. Sunter ${ }^{\mathrm{b}}$, F. Najafi ${ }^{\mathrm{b}}$, Karl K. Berggren ${ }^{\mathrm{b}}$, Vikas Anant ${ }^{\mathrm{c}}$ \\ ${ }^{a}$ IBM T.J. Watson Research Center, 1101 Kitchawan Rd, Yorktown Heights, NY, USA 10598 \\ ${ }^{\mathrm{b}}$ Research Laboratory of Electronics, MIT, 77 Massachussets Ave, Cambridge, MA, USA 02139 \\ ${ }^{\mathrm{c}}$ Photon Spot Inc., 142 W Olive Ave, Monrovia, CA, USA 91016
}

\begin{abstract}
Today Superconducting Nanowire Single-Photon Detectors (SNSPDs) are commonly used in different photon-starved applications, including testing and diagnostics of VLSI circuits. Detecting very faint signals in the near-infrared wavelength range requires not only good detection efficiency, but also very low Dark Count Rate (DCR) and jitter. For example, low noise is crucial to enable ultra-low voltage optical testing of integrated circuits. The effect of detector temperature and background thermal radiation on the noise of superconducting single-photon detectors made of $\mathrm{NbN}$ meanders is studied in this paper. It is shown that two different regimes can be identified in the DCR vs. bias current characteristics. At high bias, the dark count rate is dominated by the intrinsic noise of the detector, while at low bias current it is dominated by the detection of stray photons that get onto the SNSPD. Changing the detector temperature changes its switching current and only affects the high bias branch of the characteristics: a reduction of the DCR can be achieved by lowering the SNSPD base temperature. On the other hand, changing the temperature of the single-photon light source (e.g. the VLSI circuit under test) only affects the low bias regime: a lower target temperature leads to a smaller DCR.
\end{abstract}

Keywords: Superconducting Nanowire Single-Photon Detector (SNSPD), Near Infrared (NIR), Time-Resolved Emission (TRE), Picosecond Imaging Circuit Analysis (PICA).

\section{SINGLE-PHOTON DETECTOR METRICS}

Single-photon detectors [1] are extremely sensitive sensors capable of registering single quanta of light in the visible and/or near infrared wavelength range. Three main features characterize this kind of detectors: (i) high Detection Efficiency $(D E)$, (ii) low Dark Count Rate $(D C R)$, and (iii) accurate timing resolution (low timing jitter $\triangle t$ ). A metric often quoted for photodetectors is the Noise Equivalent Power (NEP) [2], and this has proved useful for optical power measurements. For single-photon detectors, the $N E P$ can be given by $(h v / D E) \cdot \sqrt{D C R}$, where $v$ is the photon frequency and $h$ is Plank's constant. The units of the $N E P$ are $W \cdot H z^{-1 / 2}$, and the lowest possible value of NEP is desirable. However, a typical detector (one that does not resolve photon number) does not measure optical power. Furthermore, $N E P$ does not take into account the timing performance of the detector. Hadfield therefore formulated a dimensionless figure of merit that takes all the three single-photon detector features into consideration [1], giving

$$
F O M=\frac{D E}{D C R \cdot \Delta t}
$$

This is a useful figure for Time-Correlated Single-Photon Counting (TCSPC) applications [3], as well as for optical quantum information applications [4] (e.g. for Quantum Key Distribution, QKD [5]).

\footnotetext{
* abahgat@us.ibm.com; phone 1914 945-2820; fax 1914 945-4329

$\dagger$ This work was done while the author, from Politecnico di Milano, was a summer intern at IBM T.J. Watson Research Center
}

\footnotetext{
Optical Sensing, Imaging, and Photon Counting: Nanostructured Devices and Applications, edited by Manijeh Razeghi, Dorota S. Temple, Gail J. Brown, Proc. of SPIE Vol. 9555,

95550C · @ $2015 \mathrm{SPIE} \cdot \mathrm{CCC}$ code: 0277-786X/15/\$18 · doi: 10.1117/12.2188234
} 


\section{SNSPD FEATURES}

The Superconducting Nanowire Single-Photon Detector used in this work consists of a nanowire of NbN in a meander shape [6]. The device is housed within a closed-cycle cryostat capable of cooling it down to a base temperature of about $2.7 \mathrm{~K}$. The optical coupling between the detector and the $9 \mu \mathrm{m}$ core single-mode fiber used to collect the light is obtained by means of a couple of lenses mounted on a 3 -axis piezo stage.

\subsection{Dark Count Rate, DCR}

The noise of the detector was characterized as a function of its bias current (Figure 1a). At lower bias the DCR increases slowly, while at higher bias the characteristics gets steeper. As it will be shown in the following section of the paper, the former regime is dominated by background noise, while the latter is dominated by the intrinsic noise of the SNSPD.

\subsection{System Detection Efficiency, SDE}

The overall system detection efficiency (Figure 1b) was measured as a function of the detector bias using a $1550 \mathrm{~nm}$ Continuous Wave (CW) laser. Knowing the power of the laser and the optical attenuation introduced, one is able to compute the efficiency after subtracting the dark counts from the total counts measured by the SNSPD and normalizing them to the energy of the photons. Figure $1 \mathrm{~b}$ shows that the system detection efficiency increases monotonically, until it almost saturates at bias currents close to the critical current (i.e. the current at which the detector nanowire is not superconducting anymore).

\subsection{Timing jitter, $\Delta t$}

The temporal resolution of the detector as a function of the bias current is shown in Figure 1c. It improves gradually as the bias is increased [6]. In fact, higher currents lead to voltage pulses with a bigger amplitude, thus improving the comparator threshold crossing in the front-end electronics (Figure 2).

\subsection{SNSPD Figure of Merit, FoM}

Based on the SNSPD dark count rate, system detection efficiency and timing jitter, one can compute the FoM for this particular single-photon detector using equation (1). Figure 3 shows the FoM as a function of the SNSPD bias current. One can notice how the noise is the main factor dictating the shape of the curve, due to the fact that it spans several orders of magnitudes as the bias current is changed (see Figure 1a). In order to optimize the figure of merit in (1) one has to minimize the detector noise by working at lower bias currents. However this regime of operation would typically result in longer measurements since the system detection efficiency is reduced (i.e. lower photon detection rate with the same optical signal intensity).

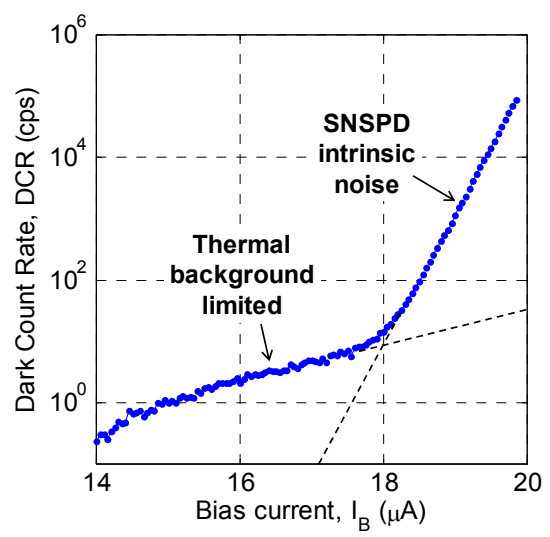

a)

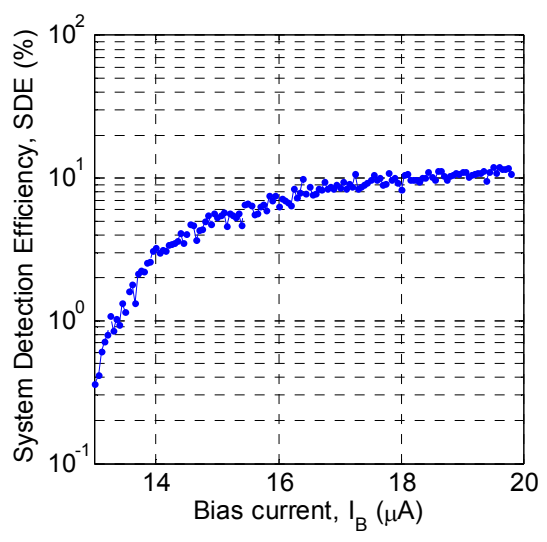

b)

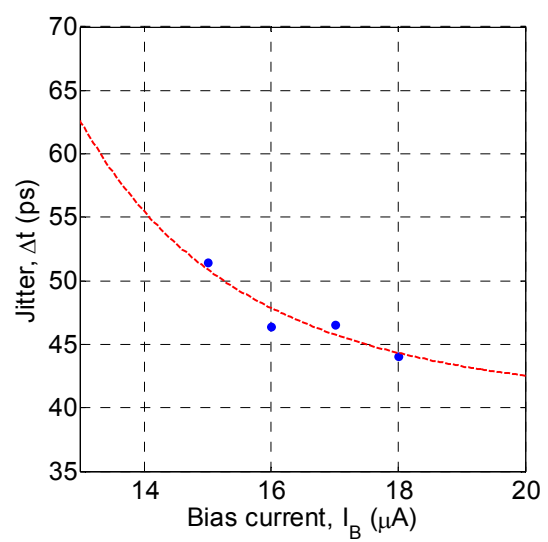

c)

Figure 1. SNSPD features at $T=2.7 \mathrm{~K}$. a) Dark count rate as a function of the bias current. Two different regions can be identified, one $\left(I_{B}<18 \mu A\right)$ where the noise is dominated by the background, and one $\left(I_{B}>18 \mu A\right)$ where it is dominated by the intrinsic noise of the detector. b) System detection efficiency $(\lambda=1550 \mathrm{~nm})$ as a function of the bias current. c) Timing jitter (Full-Width at Half-Maximum, FWHM) as a function of the bias current. 


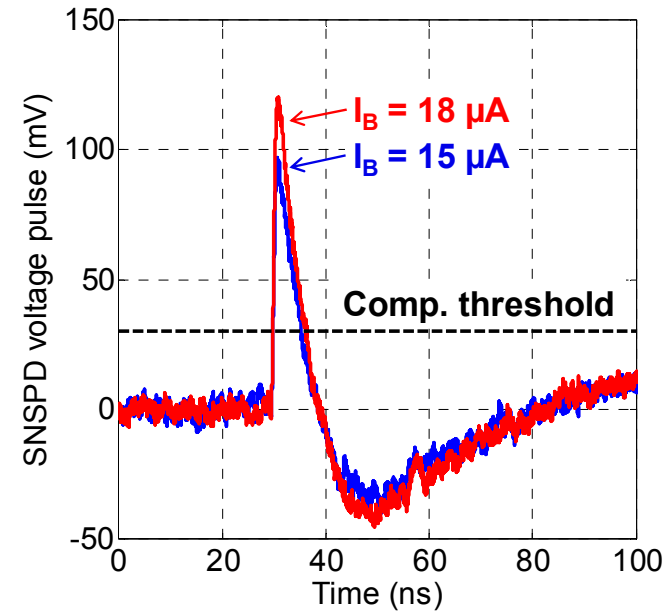

a)

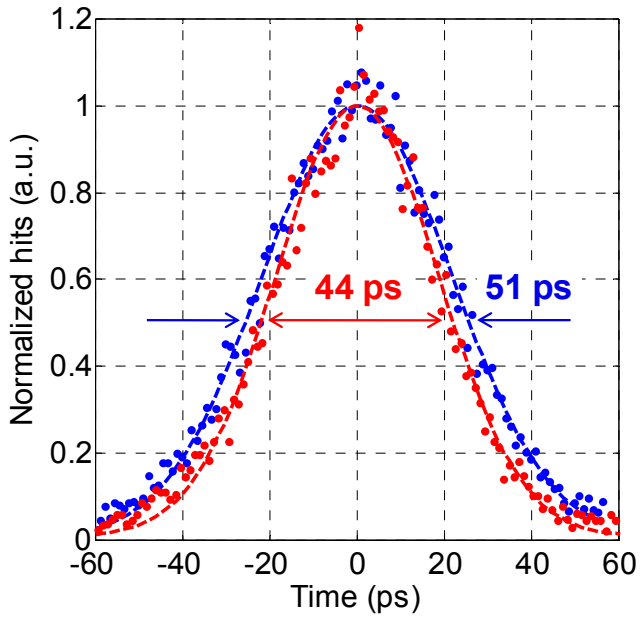

b)

Figure 2. a) SNSPD voltage pulse at the input of the front-end comparator with which the photon detection is sensed [6]. A higher bias current leads to a bigger voltage amplitude, thus reducing the electrical noise effect when crossing the threshold, and, in turn, the timing jitter, as shown in b). A high bandwidth oscilloscope $(12 \mathrm{GHz})$ was used in histogram mode to characterize the jitter of the detector (monitoring the comparator output).

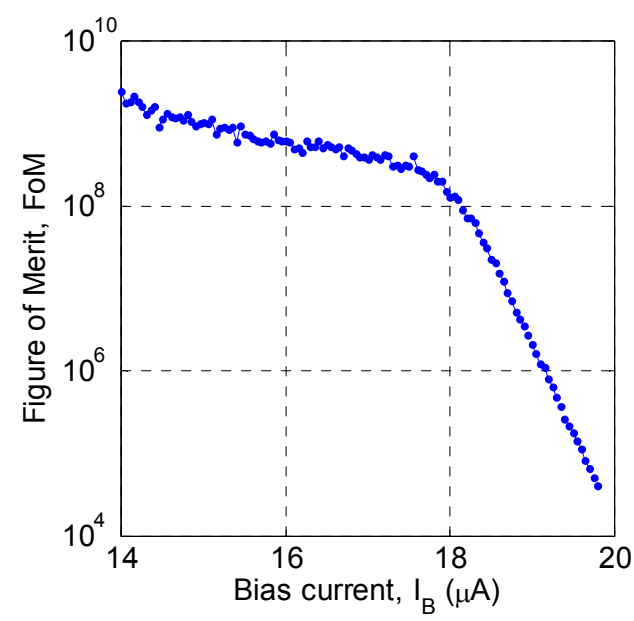

Figure 3. SNSPD figure of merit as a function of detector bias current. The main contribution to this trend is given by the dark count rate, which increases by order of magnitudes with $I_{B}$. As a result, such figure of merit is maximized at low bias, where the detector noise is limited (see Figure 1a).

\section{EFFECT OF TEMPERATURE ON DETECTOR NOISE}

\subsection{Cryostat base temperature}

In order for the SNSPD to operate, the temperature must be lower than its critical temperature. Closed-cycle liquid helium cryostats are capable of cooling the detector to temperatures lower than $4 \mathrm{~K}$. Depending on how much the detector is cooled down, its noise performance can be different. Figure 4a shows the dark count rate of the NbN SNSPD when operated at $3.4 \mathrm{~K}$ (red) or $2.7 \mathrm{~K}$ (blue). The cryostat used for these measurements was the same in both cases. By looking at the $D C R$ vs. $I_{B}$ trend one can notice that: (i) the critical current increases when operating at lower temperature $(19.8 \mu \mathrm{A} v$ v. $17.2 \mu \mathrm{A})$, (ii) the high-slope branch of the characteristics rigidly shift towards higher bias currents, and (iii) at low bias the noise remains unchanged. This suggests that only the steeper portion of the dark count rate curve 


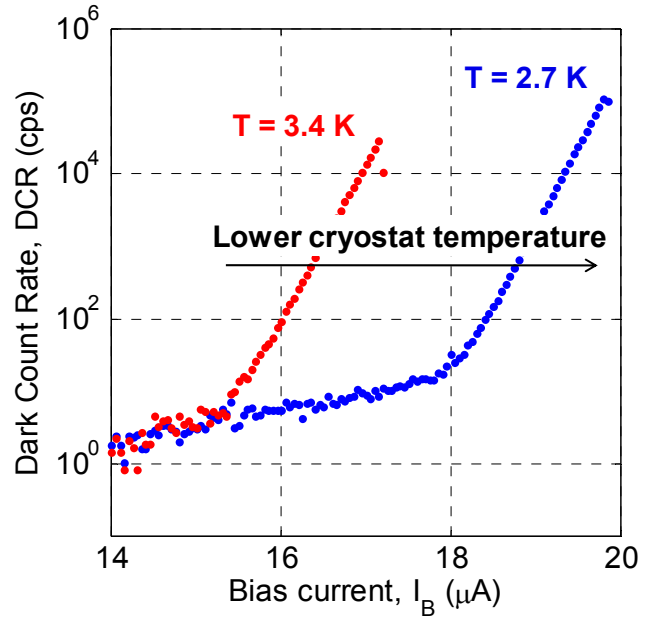

a)

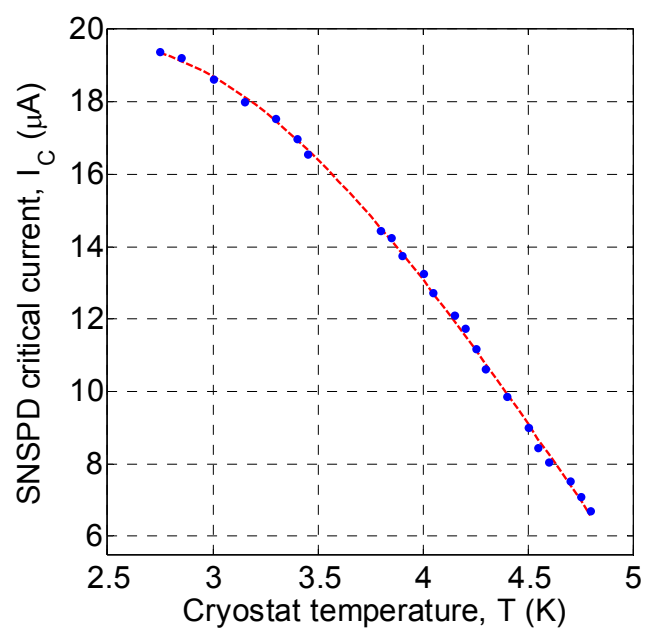

b)

Figure 4. a) Effect of cryostat temperature on dark count rate vs. bias current characteristics. Lowering the detector temperature makes its critical current increase (b) and shifts the steeper part of the DCR vs. $I_{B}$ characteristics towards the right.

depends on the actual SNSPD noise (referred to as intrinsic noise throughout the rest of this paper), while the system noise at low bias is dominated by some other mechanisms that are independent of the detector temperature. Figure $4 \mathrm{~b}$ shows the critical current measured during the cryostat cool down: at higher temperatures $I_{C}$ linearly increases as the temperature goes down, showing a sublinear dependence once the cryostat temperature goes below about $3.5 \mathrm{~K}$.

\subsection{Background thermal radiation}

If the system dark count rate was given by only the detector dark count rate, one would expect it to be lower at low bias currents. For the SNSPD used for this work, for every increase in bias of one microampere, the DCR increases by a factor of 150. In order to understand where the second regime of the noise characteristics (i.e. the flatter one) comes from, some experiments were done changing the temperature of the target from which the light/radiation was collected from.

A first set of measurements was performed by heating/cooling the single-mode fiber cap above/below ambient temperature. Note that the fiber used outside the cryostat is armored, in order to stop any ambient light. Hot/cold air was blown onto the fiber cap and the temperature was monitored using a thermocouple throughout the duration of the experiment. Figure 5a shows how the dark count rate at low bias increases when the fiber cap is heated up, while it decreases when it is cooled down. At higher bias currents, instead, the system dark count rate remains unchanged (i.e. equal to the intrinsic SNSPD noise). This result suggests that the detector is sensitive to background thermal radiation that couples into the single-mode fiber. It is interesting to note that the noise at low bias can also be reduced. A possible solution to achieve this effect would be to use a cold filter (that does not emit blackbody radiation) inside the cryostat, just in front of the detector, to block the thermal photons coming from the light source to be measured. In the case of optical testing of integrated circuits, a good cutoff wavelength for such filter would be around $2000 \mathrm{~nm}$, not to block a significant portion of the intrinsic photon emission spectrum from the circuit [7] but, at the same time, to limit the noise contribution due to blackbody radiation. If using such a solution one could get closer to the asymptote given by the SNSPD intrinsic noise, also the detector figure of merit would be consequently orders of magnitude higher in the lower bias regime (Figure 5b). Having a system that allows one to change the optical coupling between the single-mode collection fiber and the detector (through a 3-axis micropositioner stage) one can further validate that at low bias the system noise is limited by stray thermal radiation injected through the collection fiber itself. In fact, when the alignment of the SNSPD is not optimized, the dark count rate at lower bias is reduced (Figure 6). Unfortunately the alignment is not a knob the user can play with, since a worse alignment results in a reduced system detection efficiency, as well. 


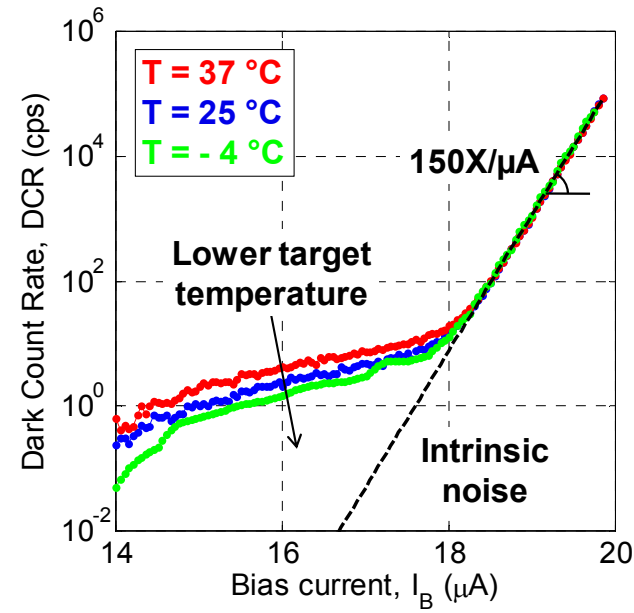

a)

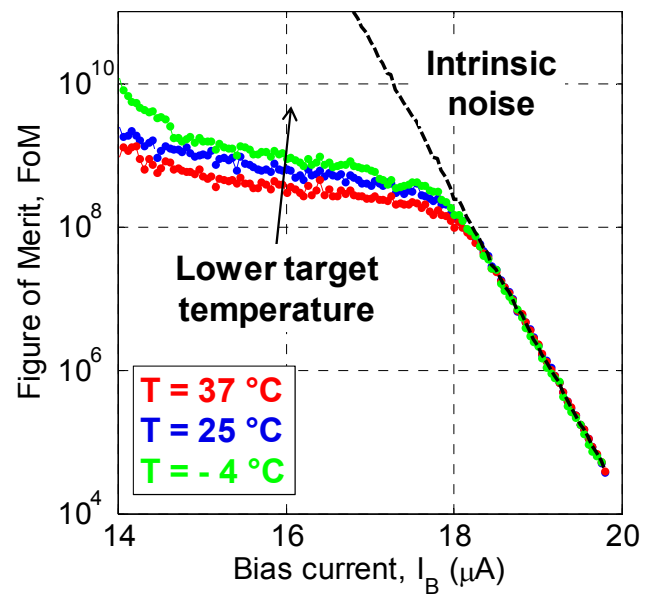

b)

Figure 5. a) System dark count rate at different fiber cap temperatures: room temperature (blue), elevated temperature (red), and below zero temperature (green). Lower target temperatures lead to a reduced dark count rate in the lower bias regime. b) The SNSPD figure of merit depends on the target temperature as well, improving when the source is colder.

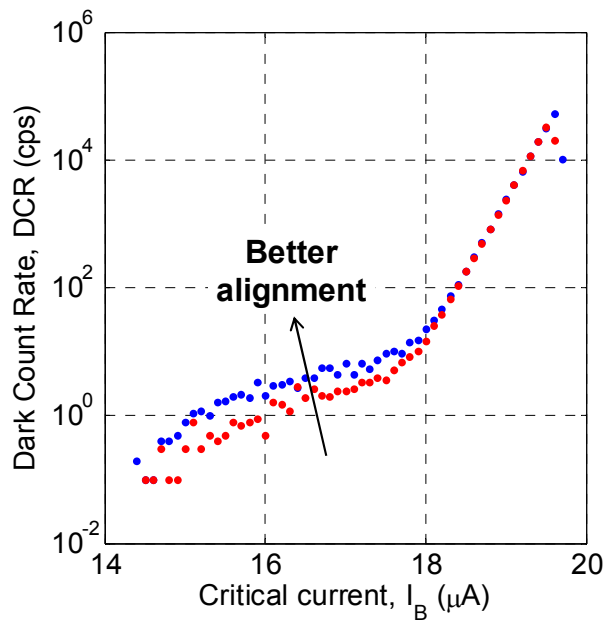

Figure 6. Effect of SNSPD alignment on system dark count rate. The fact that a better alignment leads to higher $D C R$ in the lower bias confirms that in such regime the system is limited by background thermal photons coupled to the detector through the collection fiber.

One application of the SNSPD detectors is non-invasive optical testing of integrated circuits [8]. In order to be able to measure the intrinsic optical emission from future technology nodes operating at lower supply voltages, very low noise is one of the features that a detector must have to enable such measurements [9]. When optically testing ICs, one collects the light emitted by the devices on the chip through the substrate, using microscopes with lenses that can provide adequate magnification based on the detail one wants to resolve on the device under test [10]. The effect of chip temperature on the SNSPD noise was studied by collecting light from an unpowered chip, but heated at different temperatures by means of an aluminum plate placed in contact with the die and heated using a couple of heating resistors being part of a regulated feedback loop with a thermocouple [11]. As shown in Figure 7 the effect of thermal radiation is bigger when using the 50× lens compared to the case of the high numerical aperture Solid Immersion Lens (SIL). This may be explained considering that the SIL has a smaller collection area on the chip compared to the collection area of the air-gap lens. Still, the effect of chip temperature is clear and one could cool the chip down during the measurements to mitigate such effects and keep the SNSPD noise at low levels. 


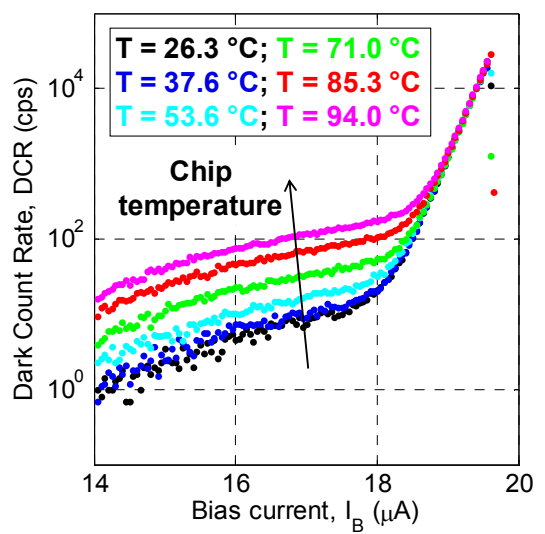

a)

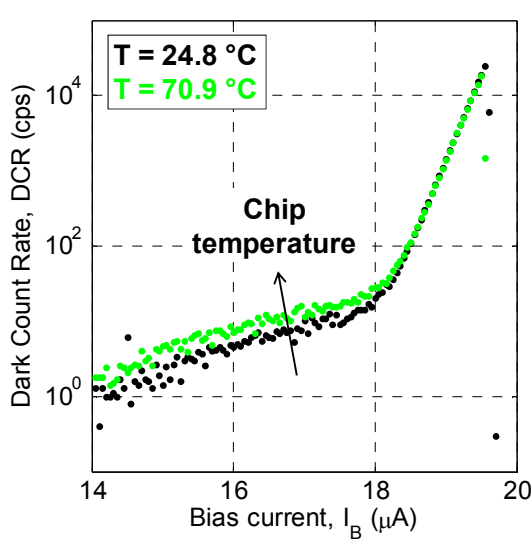

b)

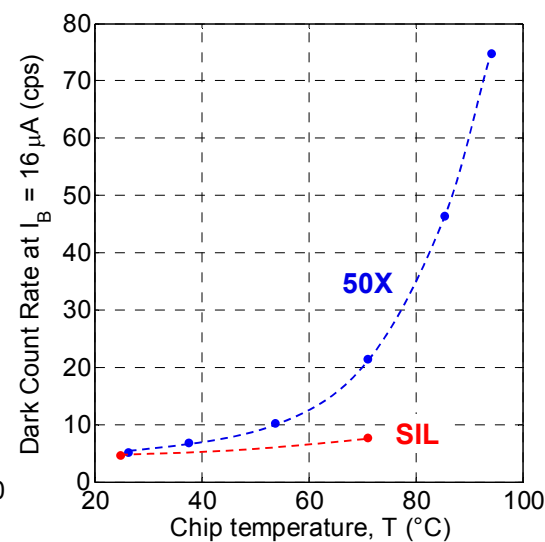

c)

Figure 7. Effect of chip temperature on system dark count rate when using the air-gap 50X lens (a) and the Solid Immersion Lens (b). The effect of thermal radiation is less critical when using the SIL (c) due to the smaller area it collects from.

\section{CONCLUSIONS}

This paper studied the effect of detector temperature and background thermal radiation on the noise of Superconducting Nanowire Single-Photon Detectors, which is one of the main features of this kind of sensor. Two regimes can be identified in the dark count rate $v s$. bias current characteristics: (i) at higher bias the noise system is dominated by the SNSPD intrinsic noise, and (ii) at lower bias it is limited by background thermal radiation that couples to the detector. It has been shown how the detector temperature only affects the high bias regime: lowering the cryostat temperature leads to an increase of the critical current and a shift of the steeper branch of the $D C R$ curve towards higher currents. On the other hand, changing the temperature of the target (i.e. the source from which one is collecting light) only affects the low bias regime. In order to improve the system noise performance at low bias, one can adopt a cold filter that blocks all the thermal photons higher than a certain cutoff wavelength. Such a solution would lead to a $D C R v s$. $I_{B}$ characteristics that is much closer to the intrinsic SNSPD noise curve at low bias current. In case of the application of the SNSPD system to non-invasive optical testing of integrated circuits, the effect of blackbody thermal radiation is mitigated when using higher magnification lenses, but cooling the chip down would be beneficial in terms of SNSPD system noise performance, and ultimately enable lower voltage measurements of ICs.

\section{AKNOWLEDGEMENTS}

The authors would like to acknowledge and thank many people from IBM T.J. Watson Research Center, DCG Systems, and MIT for useful discussions, suggestions, and support, including: Marc Taubenblatt, Mehmet Soyuer, Hervé Deslandes, Ted Lundquist, Euan Ramsay, Andrew Dane.

The project was supported by the Intelligence Advanced Research Projects Activity (IARPA) via Air Force Research Laboratory (AFRL) contract number FA8650-11-C_7105. The U.S. Government is authorized to reproduce and distribute reprints for Governmental purposes notwithstanding any copyright annotation thereon. Disclaimer: The views and conclusions contained herein are those of the authors and should not be interpreted as necessarily representing the official policies or endorsements, either expressed or implied, of IARPA, AFRL, or the U.S. Government. 


\section{REFERENCES}

[1] Hadfield, R.H., "Single-photon detectors for optical quantum information applications," Nature Photonics 3, 696-705 (2009).

[2] Donati, S., [Photodetectors: devices, circuits and applications], Prentice Hall, Ch. 3 (2000).

[3] Becker, W., [Advanced time-correlated single photon counting techniques], Springer, Ch. 2 (2005).

[4] Migdall, A., "Introduction to journal of modern optics special issue on single-photon: detectors, applications, and measurement methods," Journal of Modern Optics 51, 1265-1266 (2004).

[5] Gisin, N., Ribordy, G., Tittel, W. and Zbinden, H., "Quantum cryptography," Review of Modern Physics 74(1), 145-195 (2002).

[6] Bahgat Shehata, A. and Stellari, F., "Tuning of superconducting nanowire single-photon detector parameters for VLSI circuit testing using time-resolved emission,” Proc. SPIE 9370, Quantum Sensing and Nanophotonic Devices XII, $93702 Z$ - 93702Z-8 (2015).

[7] Bahgat Shehata, A., Stellari, F., Weger, A. and Song, P., "Modeling of transient and static components of intrinsic emission from VLSI circuits," Proc. IRPS, 4A.1.1-4A.1.7 (2014).

[8] Kash, J.A. and Tsang, J.C., "Dynamic internal testing of CMOS circuits using hot luminescence," IEEE Electron Dev. Let. 18(7), 330-332 (1997).

[9] Bahgat Shehata, A., Stellari, F., Weger, A., Song, P., Anant, V., Sunter, K., Berggren, K.K., Lundquist, T. and Ramsay, E., "Ultra-low voltage Time-Resolved Emission measurements from $32 \mathrm{~nm}$ SOI CMOS integrated circuits," Proc. ISTFA, 415-421 (2014).

[10] Bahgat Shehata, A., Stellari, F., Weger, A., Song, P., Deslandes, H., Lundquist, T. and Ramsay, E., "Novel NIR camera with extended sensitivity and low noise for photon emission microscopy of VLSI circuits," Proc. ISTFA, 6-11 (2014).

[11] Bahgat Shehata, A., Weger, A.J., Stellari, F., Song, P., Deslandes, H., Lundquist, T. and Ramsay, E., "Time-integrated photon emission as a function of temperature in $32 \mathrm{~nm}$ CMOS," Proc. IRPS, 2B.4.1-2B.4.7 (2015). 